\title{
Accumulation of allelic losses on chromosome 10 in human gliomas at recurrence
}

\author{
K Tokiyoshi, T Yoshimine, M Maruno, A K M G Muhammad, T Hayakawa
}

\begin{abstract}
Aims-To elucidate the implications of allelic loss on chromosome 10 in the malignant progression of human gliomas. Methods-Eight microsatellite loci (D10S249, D10S191, D10S210, D10S219, D10S246, D10S222, D10S221, and D10S212) were analysed for chromosomal deletions in histologically benign and malignant, including recurrent, gliomas. Of the 16 original tumours studied (two astrocytomas, nine anaplastic astrocytomas and five glioblastomas), the histological diagnosis at recurrence was anaplastic astrocytoma in six cases and glioblastoma in 10. Genomic DNA was extracted from formalin fixed, paraffin wax embedded sections. Samples of original and recurrent tumours were paired and amplified using PCR. Samples of histologically normal brain served as controls.

Results-Of the original tumours, all five glioblastomas, five $(56 \%)$ of nine anaplastic astrocytomas and none of the astrocytomas demonstrated loss of heterozygosity (LOH) on chromosome 10. Additional LOH was detected in the five cases of anaplastic astrocytoma that progressed to glioblastoma at recurrence. Additional LOH was not detected in the two cases of astrocytoma that progressed to anaplastic astrocytoma at recurrence. With the exception of one case, additional LOH was observed in the recurrent glioblastomas. Conclusion-LOH was observed at the loci of two adjacent microsatellite markers, D10S222 and D10S221 (10q23-q25), suggesting that this region on chromosome 10 is closely related to progression from anaplastic astrocytoma to glioblastoma.
\end{abstract}

(f Clin Pathol: Mol Pathol 1996;49:M218-M222)

Keywords: chromosome 10, gliomas, loss of heterozygosity, microsatellite marker, recurrence.

The presence of fairly consistent chromosomal aberrations in human gliomas suggests that such genetic changes might be involved in neoplastic transformation or malignant progression of these tumours. ${ }^{1-7}$ To date, the most frequent chromosomal changes seen in the most malignant glioma type-that is, glioblastoma, are loss of alleles on chromosome $10 .^{8-12}$ In many cases a large portion of this chromosome may be deleted..$^{13}$ These findings suggest that there is a close relation between deletions on chromosome 10 and malignant progression in gliomas. However, the loci deleted and the implications of these deletions have yet to be elucidated.

In the present study, loss of heterozygosity (LOH) was detected using multiple microsatellite markers. Special effort was made to elucidate the role of allelic losses in the malignant progression of gliomas. For this purpose, we examined paired samples of tumours obtained from the same patients at initial surgery and at recurrence.

\section{Methods}

Tissue samples were obtained from 16 patients with gliomas both at initial surgery and surgery following recurrence. Samples were fixed in $10 \%$ formalin and embedded in paraffin wax. Tumours were classified as recommended by the World Health Organisation (WHO), ${ }^{15}$ as astrocytoma in cases 1 and 2, anaplastic astrocytoma in cases 3-11 and glioblastoma in cases 12-16 at the initial surgery. At recurrence, the tumours were re-classified as anaplastic astrocytoma in cases $1-5$ and glioblastoma in cases $6-16$. The mean interval between the initial surgery and surgery for recurrence was 20.9 months (range 7-64 months).

\section{EXTRACTION OF DNA}

Paraffin wax sections, $6 \mu \mathrm{m}$ thick, were stained with haematoxylin and eosin and the areas of tumour tissue were distinguished from adjacent brain tissue by microscopic examination. Genomic DNA was extracted from one to two adjacent sections, $10 \mu \mathrm{m}$ thick. Normal brain and tumour tissue were separated carefully and processed separately for DNA extraction.

After deparaffinisation in xylene and $100 \%$ ethanol, the samples were digested in $100 \mathrm{mM}$ Tris- $\mathrm{HCl}$ (pH 8.0), 50 mM EDTA (pH 8.0) and $10 \%$ SDS containing $100 \mathrm{mg} / \mathrm{ml}$ proteinase $\mathrm{K}$ at $55^{\circ} \mathrm{C}$ overnight. Lysates were extracted twice with phenol/chloroform ( $\mathrm{pH}$ 8.0) and then twice with chloroform alone. The DNA was precipitated in 0.1 volumes of $3 \mathrm{M}$ sodium acetate $(\mathrm{pH} 5.2)$ and 2.5 volumes of $100 \%$ ethanol and resuspended in $10 \mathrm{mM}$ Tris and $1 \mathrm{mM}$ EDTA (pH 8.0).

The DNA content of samples was estimated using a spectrophotometer (UV-1200, Shimazu Corp., Kyoto, Japan). The samples were then stored at $-20^{\circ} \mathrm{C}$ pending analysis.

\section{POLYMERASE CHAIN REACTION}

Chromosome 10 polymorphisms were detected by PCR using a total of eight microsatellite markers (D10S249, D10S191, D10S210, D10S219, D10S246, D10S222, $\mathrm{D} 10 \mathrm{~S} 221$, and D10S212). The primers used 
were as suggested by Weissenbach $e t$ al ${ }^{16}$ and were purchased from Research Genetics Inc., Huntsville, Alabama, USA.

Each PCR volume $(20 \mu \mathrm{l})$ contained $200 \mathrm{ng}$ genomic DNA, $0.5 \mathrm{mM}$ primer, $200 \mathrm{mM}$ deoxynucleotide triphosphate, and 1 unit of Taq polymerase in a buffer containing $2.5 \mathrm{mM}$ Tris- $\mathrm{HCl}$ (pH 8.3), $50 \mathrm{mM} \mathrm{KCl}$ and $1.5 \mathrm{mM}$ $\mathrm{MgCl}_{2}$ (TaKaRa Syuzo Corp., Shiga, Japan). PCR conditions were as follows: $94^{\circ} \mathrm{C}$ for one minute, $53^{\circ} \mathrm{C}$ for two minutes and $72^{\circ} \mathrm{C}$ for one minute in an automated DNA thermal cycler (Sanyo Electric Corp., Osaka, Japan) for 35 cycles.

ELECTROPHORESIS AND STAINING

Ten microlitres of each PCR product were loaded onto a polyacrylamide gel cassette (Multi Gel 15/25, Daiichi Pure Chemicals, Tokyo, Japan) and subjected to electrophoresis for one hour at $40 \mathrm{~mA}$ constant current. The gels were stained with $0.5 \mu \mathrm{g} / \mathrm{ml}$ ethidium bromide and photographed using an ultraviolet transilluminator (Spectroline, TM-312A, Spectronics, Westbury, New York, USA).

\section{Results}

Figure 1 shows typical LOH at the D10S249, D10S222 and D10S221 loci in case 9. Results at initial surgery are summarised in fig 2 . Neither LOH nor homozygous deletion was observed in any of the cases of astrocytoma. Five $(55.6 \%)$ of nine cases of anaplastic astrocytoma presented with $\mathrm{LOH}$ at one to three loci: three of seven informative cases at D10S249; one of eight informative cases at D10S191; two of nine informative cases at D10S246; one of nine informative cases at D10S221; and one of nine informative cases at
D10S212. LOH was observed in all five cases of glioblastoma: one of four informative cases at D10S249; two of five informative cases at D10S210; three of five informative cases at D10S219; one of five informative cases at D10S246; two of four informative cases at D10S222; one of four informative cases at D10S221; and one of five informative cases at D10S212.

Figure 3 summarises the results of SDSPAGE analysis of all recurrent cases. Tumours in cases 1 and 2 , originally classified as astrocytoma, were re-classified as anaplastic astrocytoma at recurrence. Neither LOH nor homozygous deletion was found in these tumours. Four of nine cases were diagnosed as anaplastic astrocytoma both initially and at recurrence (cases 3-6). LOH was not detected in these tumours on either occasion. The remaining five cases of anaplastic astrocytoma were re-classified as glioblastoma at recurrence. Additional $\mathrm{LOH}$ was detected in these cases at recurrence (cases 7-11). Four of the five cases initially dignosed as glioblastoma (cases 12-16) developed additional $\mathrm{LOH}$ at recurrence (cases 12-15). Additional LOH observed in these nine cases (cases 7-15) was frequently located at the $\mathrm{D} 10 \mathrm{~S} 222$ and D10S221 loci (five of eight informative cases for each locus). Additional $\mathrm{LOH}$ was observed less frequently at D10S219 (three of nine informative cases), D10S191 (two of eight informative cases), D10S246 (two of nine informative cases), D10S249 and D10S210 (one of eight informative cases for each locus), and D10S212 (one of nine informative cases). Interestingly, of the five cases of anaplastic astrocytoma which progressed to glioblastoma, accumulation of new LOH occurred particularly at D10S222 (four instances; cases 7-10) and D10S221 (three instances; cases 7-9).

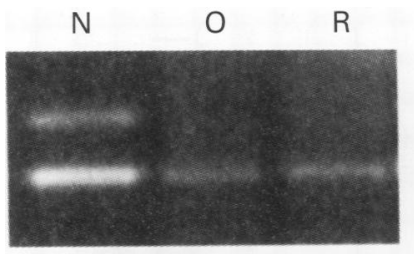

D10S249

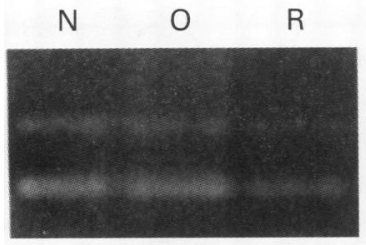

D10S219

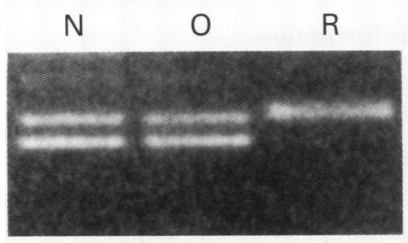

D10S221

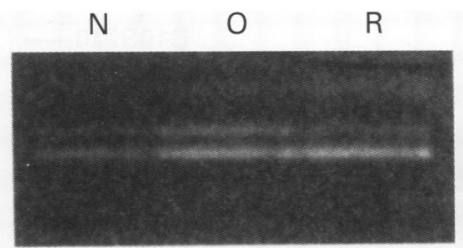

D10S191

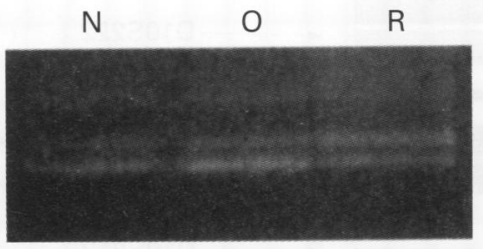

D10S246

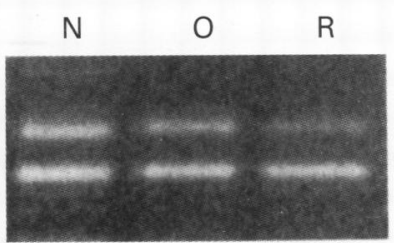

D10S212

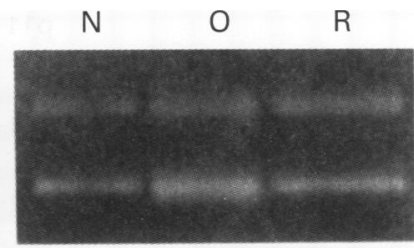

D10S210

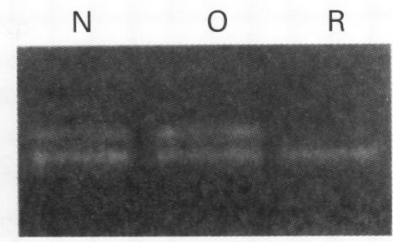

D10S222

Figure 1 An example of PCR analysis of LOH on chromosome 10 in normal brain tissue (N), original tumour (O) and recurrent tumour $(R)$ in case 9. LOH was detected at D105249 in the original tumour and at D10S249, D10S222 and D10S221 in the recurrent tumour. 


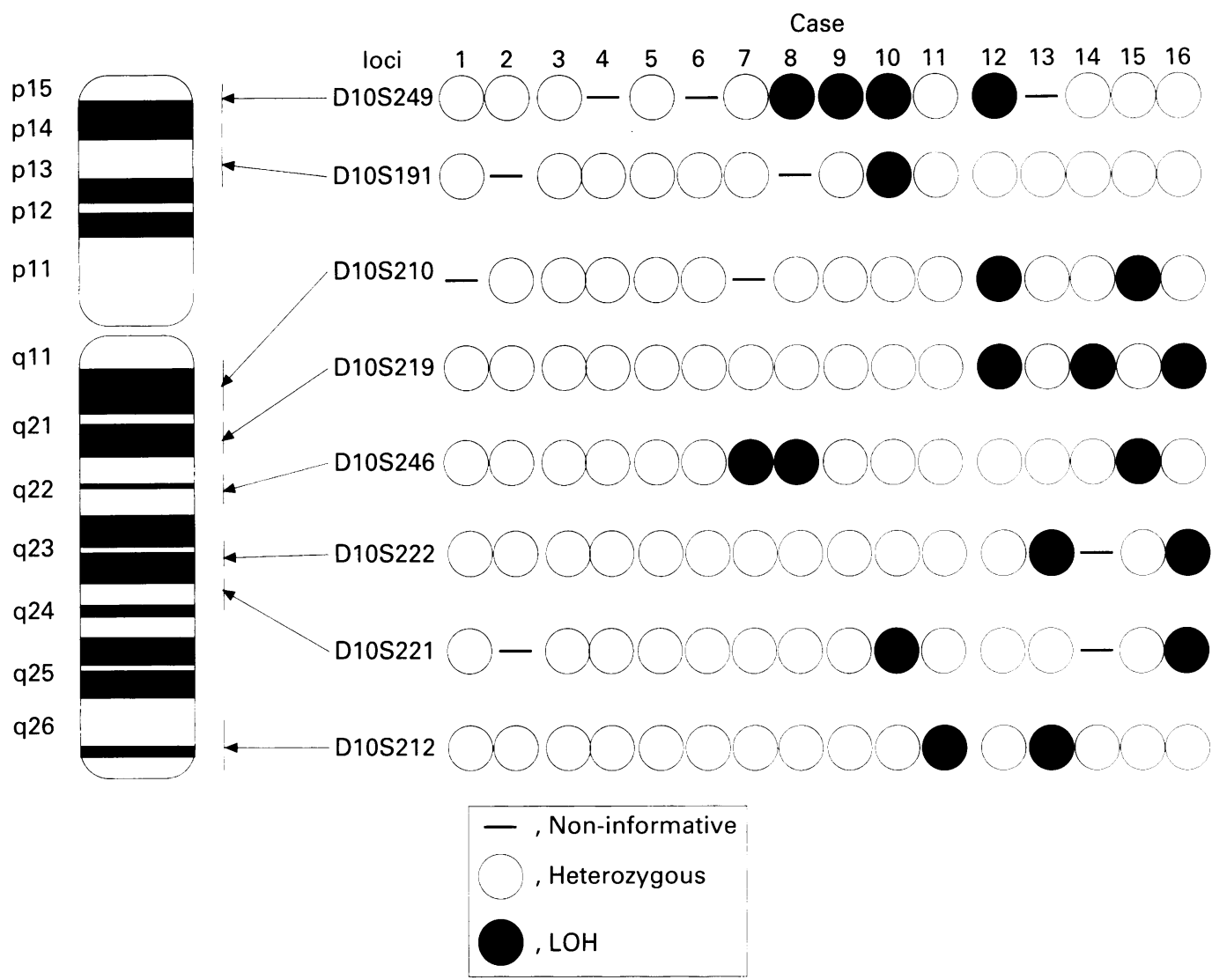

Figure 2 Summary of PCR analysis of the 16 original tumours. Cases 1 and 2 are astrocytomas, cases 3-11 are anaplastic astrocytomas, and csses $12-16$ are glioblastomas.

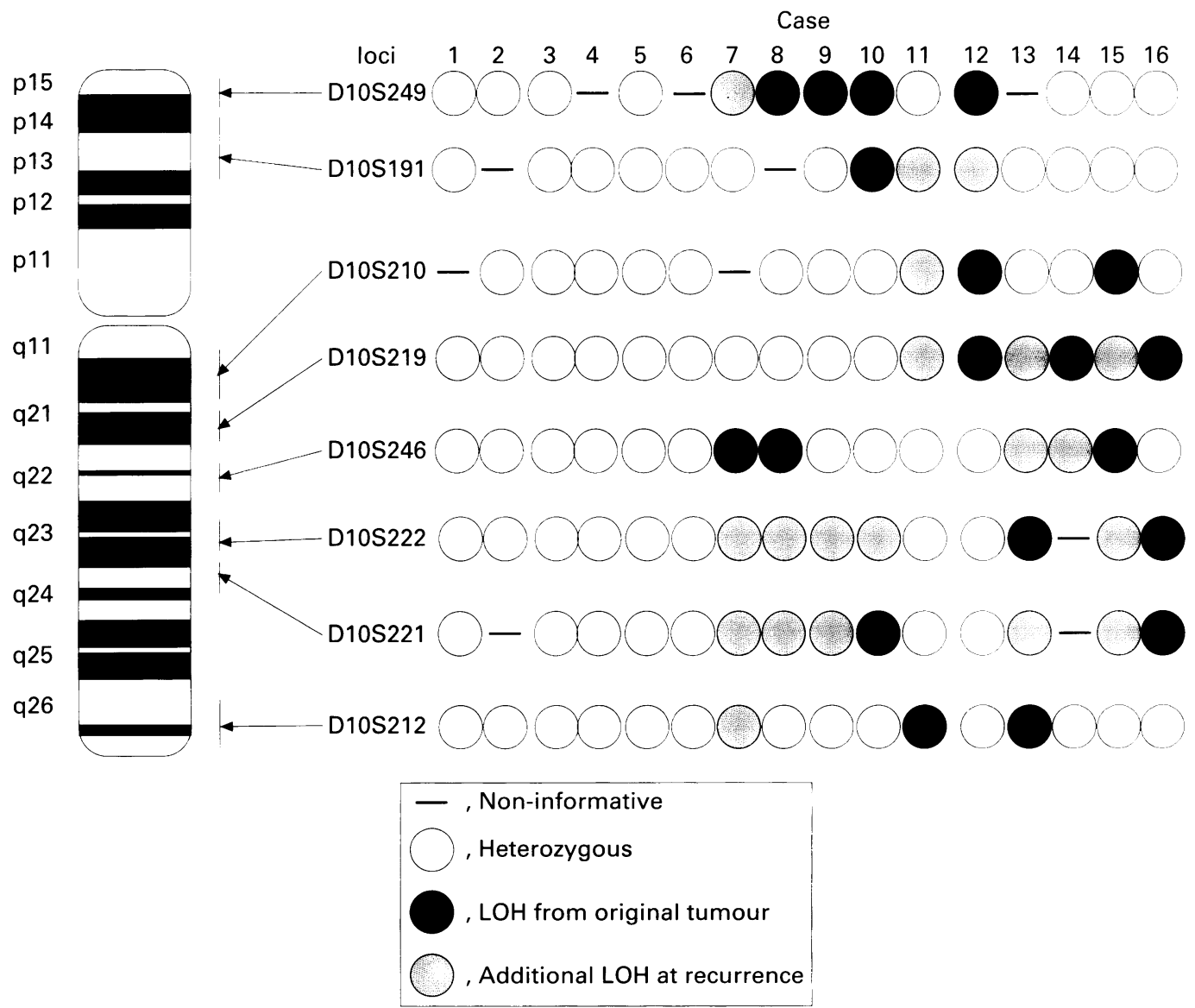

Figure 3 Summary of PCR analysis of the 16 recurrent tumours. Cases 1-6 were diagnosed as anaplastic astrocytomas, and cases 7-16 as glioblastomas at recurrence. 


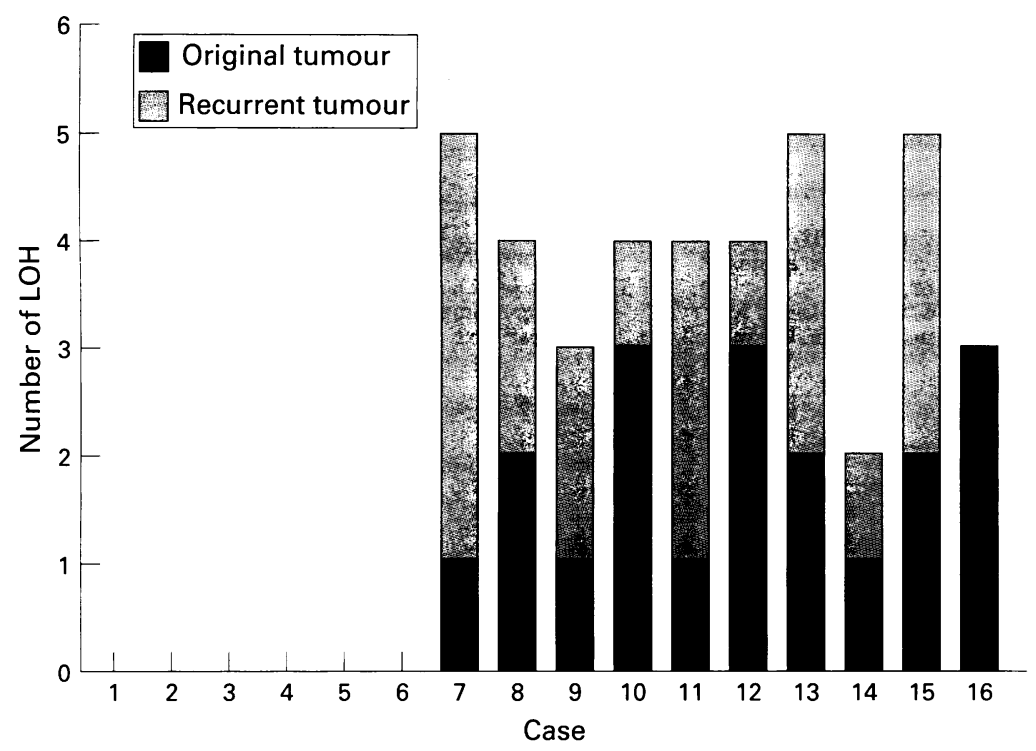

Figure 4 Number of loci with LOH detected in each case.

\section{Discussion}

In the present study, all five cases of glioblastoma and five $(56 \%)$ of nine cases of anaplastic astrocytoma had LOH on chromosome 10 at presentation. The frequency of $\mathrm{LOH}$ detected by us is higher than that found by previous investigators, who demonstrated an overall frequency of LOH of $60-95 \%$ in glioblastoma and of $12-15 \%$ in anaplastic astrocytoma. ${ }^{1}+111217-19$ Separation of the tumour tissue from the surrounding brain tissue and the use of multiple microsatellite markers in the present study may have contributed to the high frequency of abnormality detected. Neither astrocytomas showed $\mathrm{LOH}$ in the present study. The absence of $\mathrm{LOH}$ in this benign glioma is consistent with the results of many

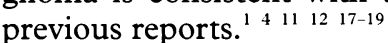

Comparison of the chromosomal changes in the tumours obtained at initial surgery and at recurrence demonstrated that all five cases originally diagnosed as anaplastic astrocytoma but which progressed to glioblastoma at recurrence gained LOH (cases 7-11). This was not the case for patients with anaplastic astrocytoma (cases 3-6). At present, there are two possible hypothetical pathways for the development of malignant glial tumours: (1) progression from low to high grade tumour; and (2) de novo development of high grade tumour with no evidence of progression from its low grade counterpart. ${ }^{11} 18$ If the former hypothesis is correct, then the five cases of anaplastic astrocytoma progressed to glioblastoma following the progression pathway (cases 7-11) and interestingly, all of these cases gained new $\mathrm{LOH}$ at recurrence. In contrast, the four cases which had retained the same histological diagnosis at recurrence, gained no new LOH (cases 3-6). These findings suggest that there is a close relation between the malignant progression of gliomas and the accumulation of $\mathrm{LOH}$ on chromosome 10 . It is possible that a tumour suppressor gene(s) is localised on this chromosome. Previous deletion mapping studies in glial tumours suggested three candidate loci for the tumour suppressor genes: at the telomeric region of the short arm of chromosome 10, at 10 cen-q23 and at $10 \mathrm{q} 23$-qter. ${ }^{13}{ }^{14} 17$ The data presented here show that those tumours which progressed from anaplastic astrocytoma to glioblastoma frequently demonstrated $\mathrm{LOH}$ at two adjacent microsatellite marker loci, D10S222 and D10S221 (80\% and $75 \%$, respectively). None of these loci were deleted in the original tumours with the exception of case 10 (D10S221). Thus, the gene(s) responsible for progression of anaplastic astrocytoma to glioblastoma may be located close to 10 q23q25.

Four of five tumours originally classified as glioblastoma also developed new $\mathrm{LOH}$ at recurrence (cases 12-15). All of these tumours possessed some $\mathrm{LOH}$ on chromosome 10 at presentation. Thus, the instability of chromosome 10 seems to increase when some $\mathrm{LOH}$ is present and accumulates further $\mathrm{LOH}$ during the course of disease (fig 4).

in conclusion, the results of the present study suggest that $\mathrm{LOH}$ on chromosome $10 \mathrm{q} 23-\mathrm{q} 25$ is closely related to the development of glioblastoma. Regional chromosomal instability seems to be especially important in the progression of anaplastic astrocytoma to glioblastoma. Further studies to identify the gene(s) located in this region and to elucidate their biological role will contribute significantly to our understanding of the oncogenesis of human gliomas.

This study was supported in part by the Grant-in-Aid for Cancer Research (4-23) from the Japanese Ministry of Health and Welfare, and Grant-in-Aid for Scientific Research (07671514) from the Japanese Ministry of Education, Science and Culture.

1 Bello MJ, Campos JM, Kusak ME, Vaquero J, Sarasa JL, Pestana A, et al. Molecular analysis of genetic abnormalities in human gliomas. Cancer Genet Cytogenet 1994;73:122-9.

2 Chung R, Whaley J, Kely N, Anderson K, Louis D, Menon $\mathrm{A}$, et al. TP53 gene mutations and $17 \mathrm{p}$ deletions in human astrocytomas. Genes Chromosom Cancer 1991;3:323-31.

3 Deimiling A, Eibl RH, Ohgaki H, Louis DN, Ammon K, Peterson I, et al. p53 mutations are associated with $17 \mathrm{p}$ allied loss in grade II and grade III astrocytoma. Cancer allied loss in grade II
Res 1992;52:2987-90.

Res 1992;52:2987-90.
4 Deimling A, Ammon K, Schoenfeld D, Wiestler OD, Seizinger BR, Louis DN. Subsets of glioblastoma multiforme defined by molecular genetic analysis. Brain Pathology 1993;3:19-26.

5 Ekstrand AJ, Sugawa N, James CD, Collins VP. Amplified and rearranged epidermal growth factor receptor genes in human gliomas reveal deletions of sequences encoding human gliomas reveal deletions of sequences encoding portions of $\mathrm{N}-$ and/or $\mathrm{C}-\mathrm{C}$
$U S A$ 1992;89:4309-13.

6 Frankel RH, Bayona W, Koslow M, Newcomb EW. p53 mutations in human malignant gliomas: comparison of loss of heterozygosity with mutation frequency. Cancer Res 1992;52:1427-33.

7 Maruno M, Yoshimine T, Muhammad AKMG, Tokiyoshi $\mathrm{K}$, Hayakawa $\mathrm{T}$. Loss of heterozygosity of microsatellite loci on chromosome $9 p$ in astrocytic tumors and its prognostic implications. 7 Neurooncol (in press)

8 Brooks-Wilson AR, Smailus DE, Weier HG, Goodfellow PJ. Human repeat element-mediated PCR: Cloning and mapping of chromosome 10 DNA markers. Genomics 1992 13:409-14

9 Karlbom AE, James CD, Boethius J, Cavenee WK, Colins VP, Nordenskjold M, et al. Loss of heterozygosity in malignant gliomas involves at least three distinct regions on chromosome 10. Hum Genet 1993;92:169-74.

10 Pierotti MA, Santoro M, Jenkins RB, Sozzi G, Bongarzone I, Greico $\mathrm{M}$, et al. Characterization of an inversion on the long arm of chromosome 10 juxtaposing D10S 170 and RET and creating the oncogenic sequence RET/PTC. Proc Natl Acad Sci USA 1992;89:1616-20.

11 Lang FF, Miller DC, Koslow M, Newcomb EW. Pathway leading to glioblastoma multiforme: a molecular analysis of genetic alterations in 65 astrocytic tumors. $\mathcal{F}$ Neurosurg of genetic alteration 
12 Pershouse MA, Stubblefield E, Hadi A, Killary AM, Yung WKA, Steck PA. Analysis of the functional role of hromosome 10 loss in human glioblastomas. Cancer Res 993;53:5043-50.

13 Ransom DT, Ritland SR, Moertal CA, Dahl RJ, O'Fallon $\mathrm{R}$, Scheithauer BW, et al. Correlation of cytogenetic analysis and loss of heterozygosity studies in huma diffuse astrocytomas and mixed oligo-astrocytomas. Gene Chromosom Cancer 1992;5:357-74.

14 Rasheed BK, Fuller GN, Friedman AH, Bigner DD, Bigner $\mathrm{SH}$. Loss of heterozygosity for $10 \mathrm{q}$ loci in human gliomas. Genes Chromosom Cancer 1992;5:75-82.

15 Kleihues $\mathrm{P}$, Burger SH, Scheithauer BW. In: Histological typing of tumors of the central nervous system. 2nd edn. Berlin:
Springer-Verlag, 1993:11-14

16 Weissenbach J, Gyapay G, Dib C, Vignal A, Morissette J, Millasseau P, et al. A second-generation linkage map of the human genome. Nature 1992;359:794-801.

17 Fults D, Brockmeyer D, Tullous MW, Pedone CA, Caethon RM. p53 mutation and loss of heterozygosity on chromosomes 17 and 10 during human astrocytoma progression. Somes 17 and 10 during hum
Cancer Res 1992;52:674-9.

18 Leon SP, Zhu J, Black PM. Genetic aberrations in human brain tumor. Neurosurgery 1994;34:708-22.

19 Ye Z, Wu JK, Darras BT. Loss of heterozygosity for alleles on chromosome 10 in human brain tumors. Neurol Res 1993;15:59-62. 